\title{
Performance considerations for continuous-wave and pulsed laser line scan (LLS) imaging systems
}

\author{
F. M. Caimi \\ f.m.caimi@ieee.org
}

\section{F. R. Dalgleish}

\author{
Ocean Visibility and Optics Laboratory, Harbor Branch Oceanographic Institute, \\ Florida Atlantic University, Fort Pierce, FL, 34946, USA \\ Ocean Visibility and Optics Laboratory, Harbor Branch Oceanographic Institute, \\ Florida Atlantic University, Fort Pierce, FL, 34946, USA
}

Extended range underwater optical imaging techniques can be classified into one of two broad categories; those which use synchronously scanned narrow source and receiver paths to restrict both back-scattered and forward-scattered light reaching the receiver (continuouswave laser line scan); and those which use pulsed sources and time-gating to remove back-scatter noise (pulsed laser line scan and pulse-gated laser line scan). Laser line scan systems are observed to perform at up to 5 to 6 optical attenuation lengths, but greater standoff distances are desirable for seabed imaging using the growing fleet of autonomous underwater vehicles (AUVs). Currently, a moderate physical separation between laser and receiver is necessary to reject near-field multiple back-scatter, which imposes restrictions on AUV miniaturization. Recent experiments and theoretical modeling reveal that significant imaging improvements are possible over the existing continuous-wave laser line scan systems (LLS), by using a pulsed-gated version of the LLS (PG-LLS). However, the use of such a technique has a greater advantage in reducing the overall form factor over conventional LLS imaging system, as well as providing greater depth-of-field. In this paper, we present experimental results comparing both LLS and PG-LLS systems for several source-receiver separations and standoff distances. These results compare favorably with images obtained from validated LLS image simulation tools, and indicate the potential for reducing the source-receiver separation and therefore the system size. [DOI: 10.2971/jeos.2010.10020S]

Keywords: underwater imaging, scattering media, lidar, light scattering, radiative transfer

\section{INTRODUCTION}

Current needs for advanced undersea imaging systems include search and rescue operations, military surveillance, offshore oil pipeline inspection, and scientific observation. These needs have partially been served by acoustic systems such as sonar and related imaging systems, which operate over distances in excess of tens of meters. These systems however, are limited in image resolution at longer standoff distances due to the highly dispersive propagation characteristics of seawater at shorter acoustic excitation wavelengths.

Conversely, specialized optical imaging systems can provide higher resolution suited for more robust object identification, but their use has been limited by hardware availability, platform size and availability necessary for operation, and limitations imposed by the system geometry.

Laser line scan (LLS) underwater imaging is a serial imaging technique which involves the optical scanning of a narrow instantaneous field of view (IFOV) receiver in a synchronous fashion with a highly collimated laser source over a wide swath. It is widely regarded as the optimal technology for extended range underwater optical imaging, with up to 6 beam attenuation lengths ${ }^{1}$ achievable in turbid seawater [1]-[4]. These imagers, which typically utilize moderate-

\footnotetext{
${ }^{1}$ One beam attenuation length is the reciprocal of the beam attenuation coefficient, $c\left(\mathrm{~m}^{-1}\right)$. Number of attenuation lengths is the physical target range multiplied by $c$.
}

power green continuous wave $(\mathrm{CW})$ lasers, require an adequate laser-receiver separation to reduce near-field multiple backscatter that emanates from the non-overlapping volume defined by the laser beam spread and enters the receiver field-of-view. Systems therefore occupy a large linear footprint which makes them unsuitable for modern unmanned underwater platforms such as the man-portable autonomous underwater vehicle (AUV).

As the source-receiver separation is reduced, elastically scattered photons arriving from scattering centers in the intervening media become larger in number than desired photons returning to the receiver from the object plane or target. In order to create an image that contains reflectance information from the object plane, the detection method must differentiate scattered photons from those arriving from the object. Well known techniques for achieving this result include time gating, where the detection system is timed to be responsive to the arrival of the object photons after most of the nearby scattered photons have arrived. These methods also have the potential to determine bathymetry from the travel time of the light pulses, and as is the intent of this paper to show, pulsed-gated imager architectures can provide a more compact LLS implementation with a reduced laser-receiver separation and equivalent performance to larger CW-LLS systems. Such techniques can allow separation of the target and scattering volume return 
signals, thereby increasing the imaging range possible under certain conditions.

Previous configurations of time gated techniques have been demonstrated using spatially broadened laser pulses and precise gated, intensified cameras, with results indicating imaging performance beyond 6 beam attenuation lengths [4, 6][10]. However, employment of wide-angle array-style detectors, allows susceptibility to image degradation from multiple forward scatter in very turbid water, even when extremely short gate times are used.

Recent simulation [11, 12] and experimental work [13] has shown that in scattering dominant waters, the PG-LLS will improve the achievable image contrast over the CW-LLS, as well as allow for more compact system implementations.

In order to alleviate the issues associated with non-coherent signal detection, coherent processing using a modulated signal was suggested, with the supposition that the scattered photons arriving separately and at different times would produce a largely non-coherent signal compared with those photons arriving at nearly the same time from the object or target [15]. Counter arguments have suggested that forward scatter spatial broadening in the outgoing beam with standoff, and consequent expansion of the point spread function which is spatially convolved with the image, is a major limiting factor to achieving significantly larger increases in contrast at limiting standoff distances [15, 18]. Selection of the modulation frequency is critical and experiments are underway in the research community to determine what gains are realizable. Recent implementations of coherent detection suggest that intensity modulation of a CW laser with coherent processing at the detection stage [27] offers some promise in improving contrast and providing bathymetric imaging capabilities from modulation phase information, but in the presence of high levels of backscatter, the shot noise being generated in the receiver governs the contrast limit $[5,27]$.

This paper utilizes a recently developed radiative transfer model [14] that incorporates both system hardware and water optical property parameters, and has been developed to aid in performance prediction and evaluation of alternative design parameters for the LLS class of underwater imagers. The laser pulse time history model evaluates the contributions from both volume backscatter and target reflections to predict the photon flux arriving at the receiver as a function of time. The model includes the option to use either a semi-analytical small-angle scattering approximation or a Monte Carlo code. The image simulation model also includes shot noise computation [14].

Having evaluated the accuracy of the backscatter time history model to predict the magnitude and timing of backscatter computation, the authors present laser line scan (LLS) image simulation results, to consider the relative merits of using either continuous wave (CW) or pulsed laser sources, as a function of source to receiver separation in order to analyze the expected imaging performance of future LLS payloads sized to be compatible with three common form factor autonomous underwater vehicles. For each payload configuration using re- alistic stand-off distances and platform velocities, four main effects are examined:

1. Effects of water beam attenuation coefficient and scattering on image performance

2. Depth-of-Field (DOF) effects on simulated images

3. Dependence of image quality (contrast) on laser power

4. Laser beam divergence effects on image resolution

The outcome of this work is intended to provide a better understanding of the optimal system configuration for LLS class of imagers for a different source-receiver separations and illustrates trends in the physical performance of such systems.

\section{TIME DEPENDENT BACKSCATTER MODEL}

The underwater lidar model calculates the time-dependent return due to volume backscatter and surface reflections in the object plane [14]. The model incorporates a source consisting of a Gaussian beam with selectable divergence angle, a receiver aperture with selectable angular and spatial aperture. The source and receiver are spatially displaced but with coincident centers of field in the object plane. The source radiance and the spatio-angular response pattern of the receiver aperture may be non-axisymmetric. The optical properties of the medium may be chosen to vary in the direction of beam propagation, but in this study are spatially constant. The model computes the temporal impulse response for the system in the prescribed environment which is then convolved with the output pulse shape to obtain the received signal.

Optical paths are limited to multiple small-angle forward scattering and a single large-angle scattering event in the backward direction [17]. The numerical formulation segments the optical medium into "slabs" of finite thickness $(d z)$ lying perpendicular to the direction of beam propagation. The return from each slab is treated in much the same way as the return from a flat, solid surface; that is, by convolving the system's spatial impulse response with the uniform reflectivity of the slab. The spatial impulse response is computed based on the solution to the radiative transfer equation under the smallangle scattering approximation (RTE/SAA), as described by Dolin [19], and with no further simplifying assumptions. The flux reflectance of a slab at distance $z$ along the beam axis is $R=\pi d z \beta_{\pi}(z)$, where $\beta_{\pi}$ is the backscatter coefficient.

A semi-analytical approach is employed that reduces the general problem for each slab to at most a few one-dimensional integrals. In the special case of axisymmetric apertures this problem reduces to a single one-dimensional integral in accord with the developments in Mertens \& Replogle [21] and Korshunov [22], for example. Conceptually, the model evaluated here has much in common with models presented by Dolin \& Saval'yev [20], Mertens \& Replogle [21], Zege et 


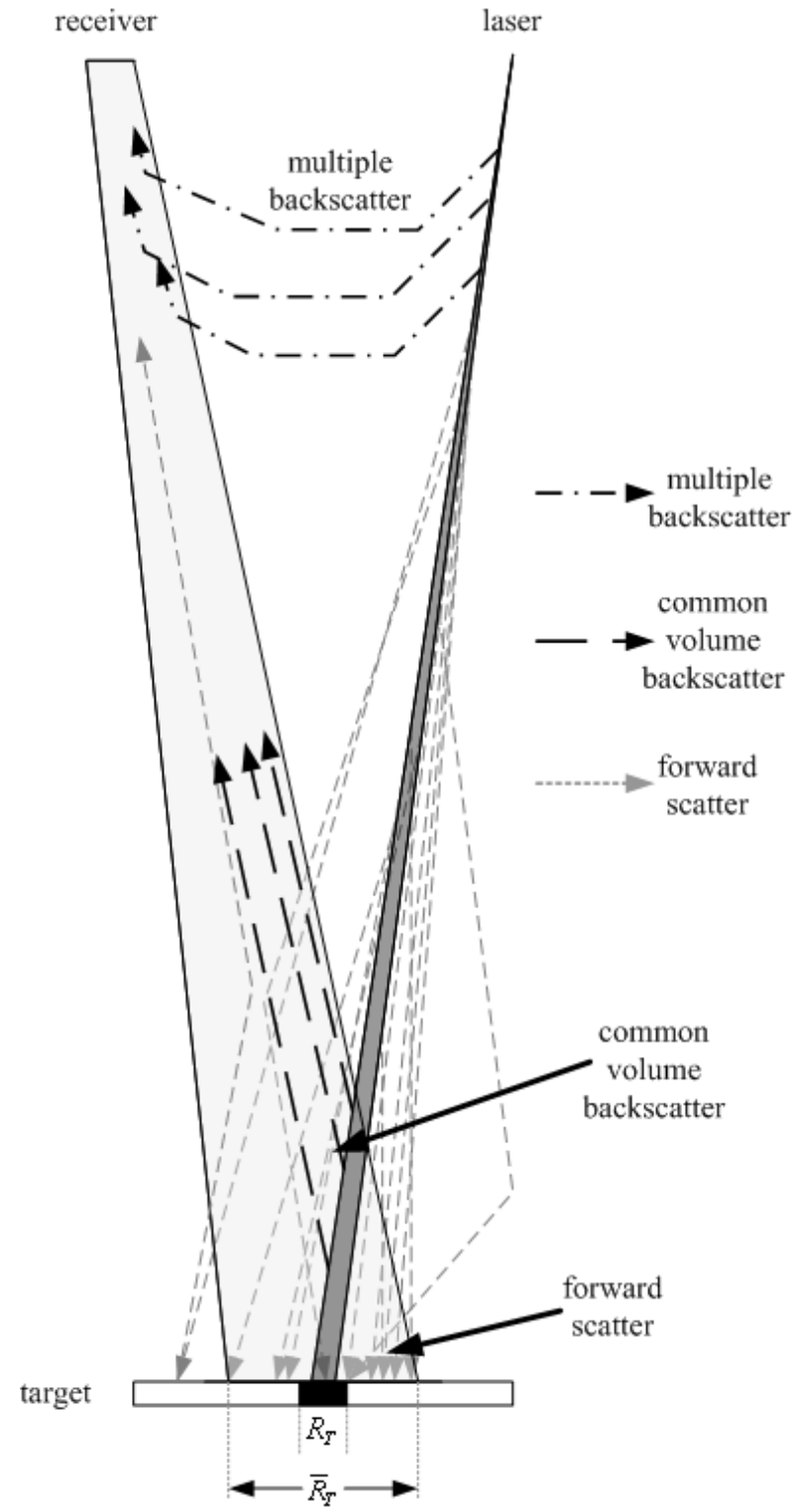

FIG. 1 Representative geometry for the LLS and PG-LLS imaging systems, showing laser, target, and receiver field-of-view.

al. [23], Katsev et al. [24], and Korshunov [22]. The main differences are that the present formulation uses the full RTE/SAA solutions and that the numerical approach greatly reduces the computational burden in the general, asymmetric aperture cases. The present model has been rigorously verified using Monte Carlo simulations and has shown excellent agreement in resolving the return from the multiple-scatter and common volume backscatter regions $[25,26]$.

The geometry representative of an LLS imaging system as modeled is shown in Figure 1. The laser is shown with finite but small angular divergence, along with target, and receiver field-of-view. Multiple scatter, common volume backscatter, and forward scatter regimes are shown.

\section{BACKSCATTER RESULTS}

The model is now used for illustration of the relative detected temporal signals for two beam attenuation lengths (one large and one small) at a fixed standoff distance. Target and com-

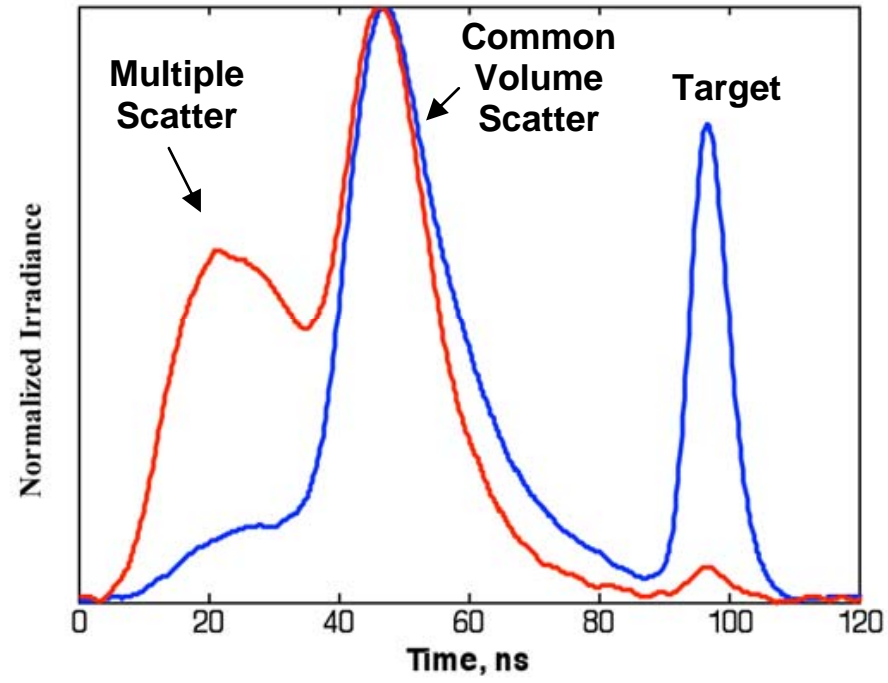

FIG. 2 Representative normalized laser pulse time history detected signal amplitude for two beam attenuation coefficients (large and small). Signals are normalized to common volume scatter peaks. At the higher beam attenuation (red), the multiple scatter peak is larger and the target return is smaller.

mon volume returns are shown for a typical laser pulse as shown in Figure 2. Light travels at nearly $22.5 \mathrm{~cm} / \mathrm{ns}$ in water or $4.4 \mathrm{~m} / \mathrm{ns}$, so figure horizontal axes are scaled in time rather than distance units. Starting from time $t=0$, the first broad return is due to multiple scattering (MS) from particles near the viewport, where the laser energy is greatest. This is followed by the narrower return from the common volume $(\mathrm{CV})$, defined by the intersection of the receiver field-of-view with the spatially spread laser beam. The last peak is due to the reflection from the target in the object plane. The CV return peak occurs close to the onset of the $\mathrm{CV}$ region since the intensity of the laser pulse falls off exponentially as it transits the CV. At larger beam attenuation, the MS return is significant and overlaps the CV return, shifting the apparent position of the $\mathrm{CV}$ peak to the left on the time axis. In the discussion that follows, we consider the effect of changing each experimental variable on the MS and CV returns.

The effects of changing the source-receiver (S-R) separation are shown in Figure 3 for the case of $10 \mathrm{mR}$ receiver angular aperture and a beam attenuation coefficient $c=0.25$. As the S-R separation is decreased for a given aperture and turbidity, the onset of both the MS and CV should begin earlier. As shown, the $83 \mathrm{~mm}$ S-R separation is most susceptible to multiple scatter. The target has been removed in Figures 3 through 8 to illustrate the system behavior only to optical property and geometric variations.

As the angular aperture is increased from $10 \mathrm{mR}$ to $30 \mathrm{mR}$ for a given S-R separation, pointing angle, and optical attenuation, we expect the onset of both the MS and CV returns to begin sooner, and for the relative detected signal amplitude to increase (see Figure 4).

Referring to Figure 1 and considering receiver pointing angles increasing from $-6 \mathrm{mR}$ to $0 \mathrm{mR}$ to $+6 \mathrm{mR}$ for a given aperture, $\mathrm{S}-\mathrm{R}$ separation, and attenuation coefficient, we expect the on- 


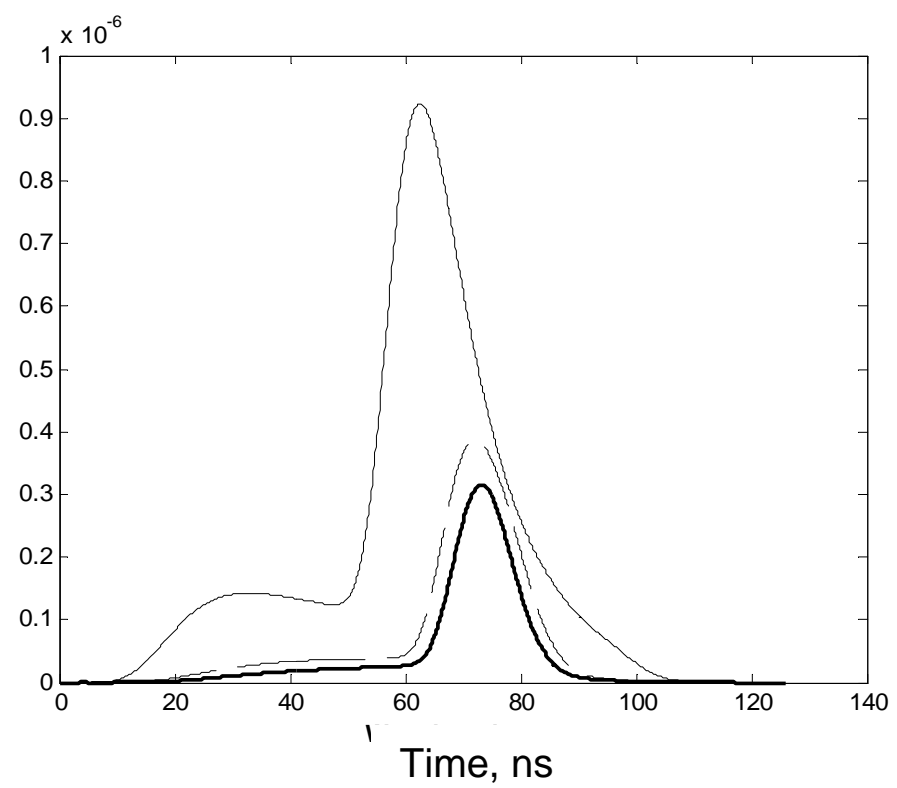

FIG. 3 Modeled backscatter time history detected amplitude signal (arbitrary linear scale) for source-receiver separations of $83 \mathrm{~mm}$ (solid line), $250 \mathrm{~mm}$ (dashed line) and $376 \mathrm{~mm}$ (thick line). Receiver aperture $=10 \mathrm{mR}$, beam attenuation $c=0.25 \mathrm{~m}^{-1}$.

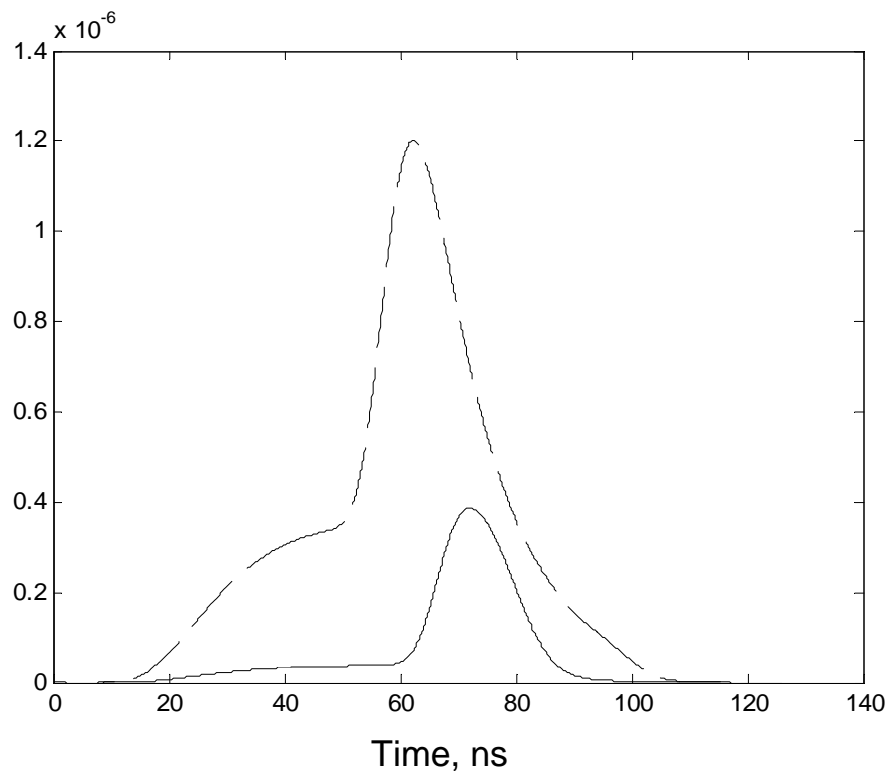

FIC. 4 Modeled backscatter time-history detected amplitude signal (arbitrary linear scale) for receiver apertures of $10 \mathrm{mR}$ (solid line) and $30 \mathrm{mR}$ (dashed line). Sourcereceiver separation $250 \mathrm{~mm}, c=0.25 \mathrm{~m}^{-1}$.

set of both the MS and CV returns to begin sooner, and for the amplitude to increase (see Figure 5).

As the beam attenuation is increased for a given aperture, $\mathrm{S}$ $\mathrm{R}$ separation, and pointing angle we expect the MS return to begin sooner and become larger. The CV signal peak should remain fixed temporally and should decrease in amplitude, as more of the energy would have been removed from the beam before it entered the CV (see Figure 6).

To illustrate the agreement of the simulation model with experimental results, we conducted measurements of detected signals using a telecentric optical system with adjustable angular aperture (30 $\mathrm{mR}$ in this case), which was in turn coupled

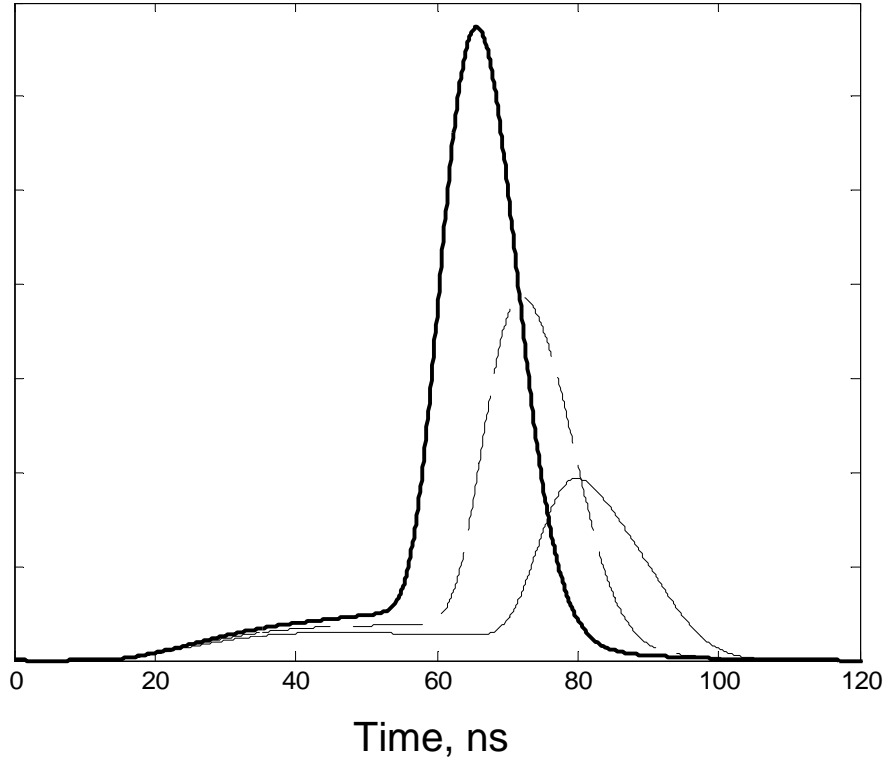

FIG. 5 Modeled backscatter time-history detected signal amplitude (arbitrary linear scale) for receiver pointing angles of $-6 \mathrm{mR}$ (solid line), o $\mathrm{mR}$ (dashed line) and $+6 \mathrm{mR}$ (thick line). Source-receiver separation $250 \mathrm{~mm}$, beam attenuation $c=0.25 \mathrm{~m}^{-1}$, receiver aperture $=10 \mathrm{mR}$.

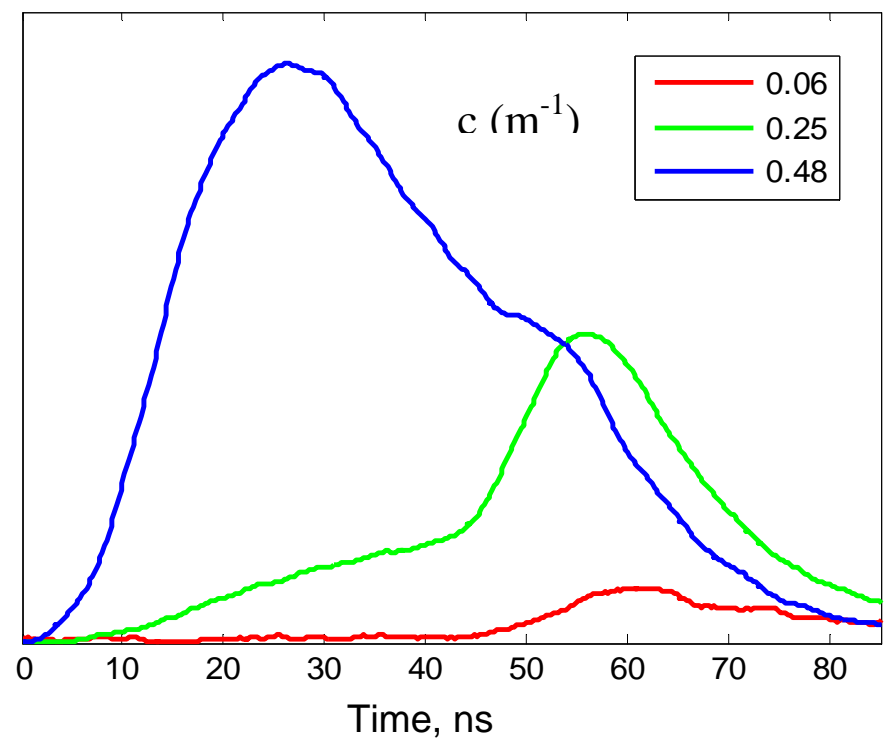

FIG. 6 Backscatter time-history relative detected signal amplitude (arbitrary linear scale) for beam attenuation coefficients of $c=0.06 \mathrm{~m}^{-1}$ (red), $0.25 \mathrm{~m}^{-1}$ (green) and $0.48 \mathrm{~m}^{-1}$ (blue). Source-receiver separation $250 \mathrm{~mm}$, receiver aperture $=30 \mathrm{mR}$.

to a high speed photomultiplier tube. The object plane was removed for this comparison. The laser source used produced $5 \mu \mathrm{J}$ pulses at $532 \mathrm{~nm}$ with FWHM pulse duration of $6.5 \mathrm{~ns}$.

Results shown in Figure 7 are generally in good agreement, and show the onset of multiple scatter as the attenuation coefficient in increased from $c=0.08 \mathrm{~m}^{-1}$ to $c=0.25 \mathrm{~m}^{-1}$. The scale is relative amplitude, linear, normalized to peak signal. The source-receiver separation (S-R) is $376 \mathrm{~mm}$, which is considered large for man-portable AUV usage.

Comparative results are shown in Figure 8 but with a reduced source-receiver separation of $250 \mathrm{~mm}$. For the smaller attenuation coefficient $\left(c=0.06 \mathrm{~m}^{-1}\right)$, the detected signal consists 

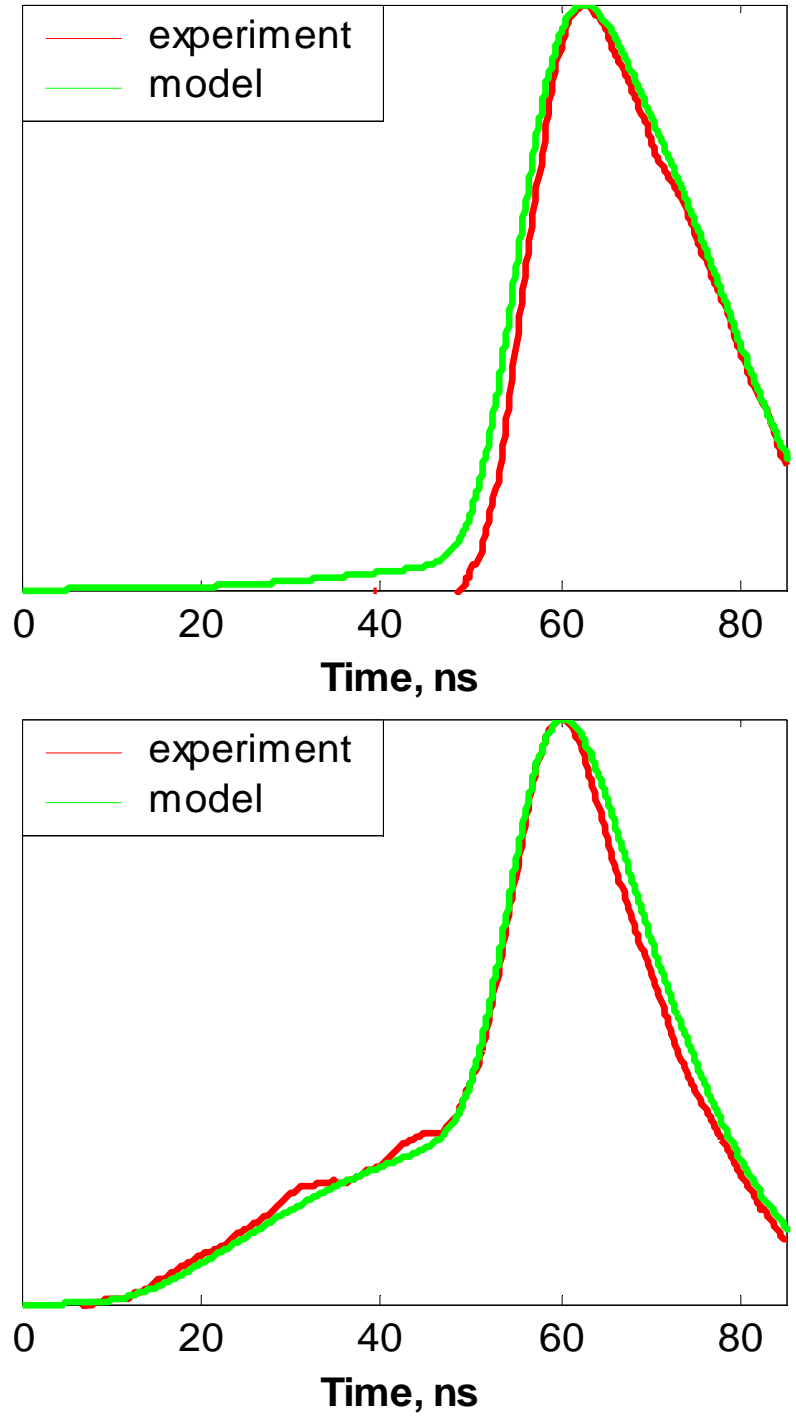

FIG. 7 Graphs of the experimental backscatter data (red) and model results (green) on arbitrary linear scale. Top: attenuation coefficient $c=0.08 \mathrm{~m}^{-1}$, S-R separation $=376 \mathrm{~mm}$, angular aperture $=30 \mathrm{mR}$. Bottom: $c=0.25 \mathrm{~m}^{-1}, \mathrm{~S}-\mathrm{R}=376 \mathrm{~mm}$, aperture $=30 \mathrm{mR}$.

mostly of backscatter. The case for $c=0.25 \mathrm{~m}^{-1}$ is directly comparable to Figure 7, and illustrates a significant contribution from multiply scattered photons from $10 \mathrm{~ns}$ to $40 \mathrm{~ns}$ round trip delay. This effect that is problematic for the CW-LLS system at shorter S-R distances, suggesting the use of a gated detector configuration.

\section{FORWARD SCATTER MODEL AND EXPERIMENT COMPARISON}

The laser spatial profile is subject to near forward angle scattering and therefore exhibits non-Gaussian spreading with distance of propagation. This effect can be described by a medium point spread function that is a function of several parameters, including laser divergence angle, scattering phase function in the near forward angular region, and distance to the plane of measurement (object plane). It is not our intent to produce a rigorous description of this effect, but rather to illustrate that the small angle approximation used in the simulation model is in good agreement with Monte-Carlo and ex-
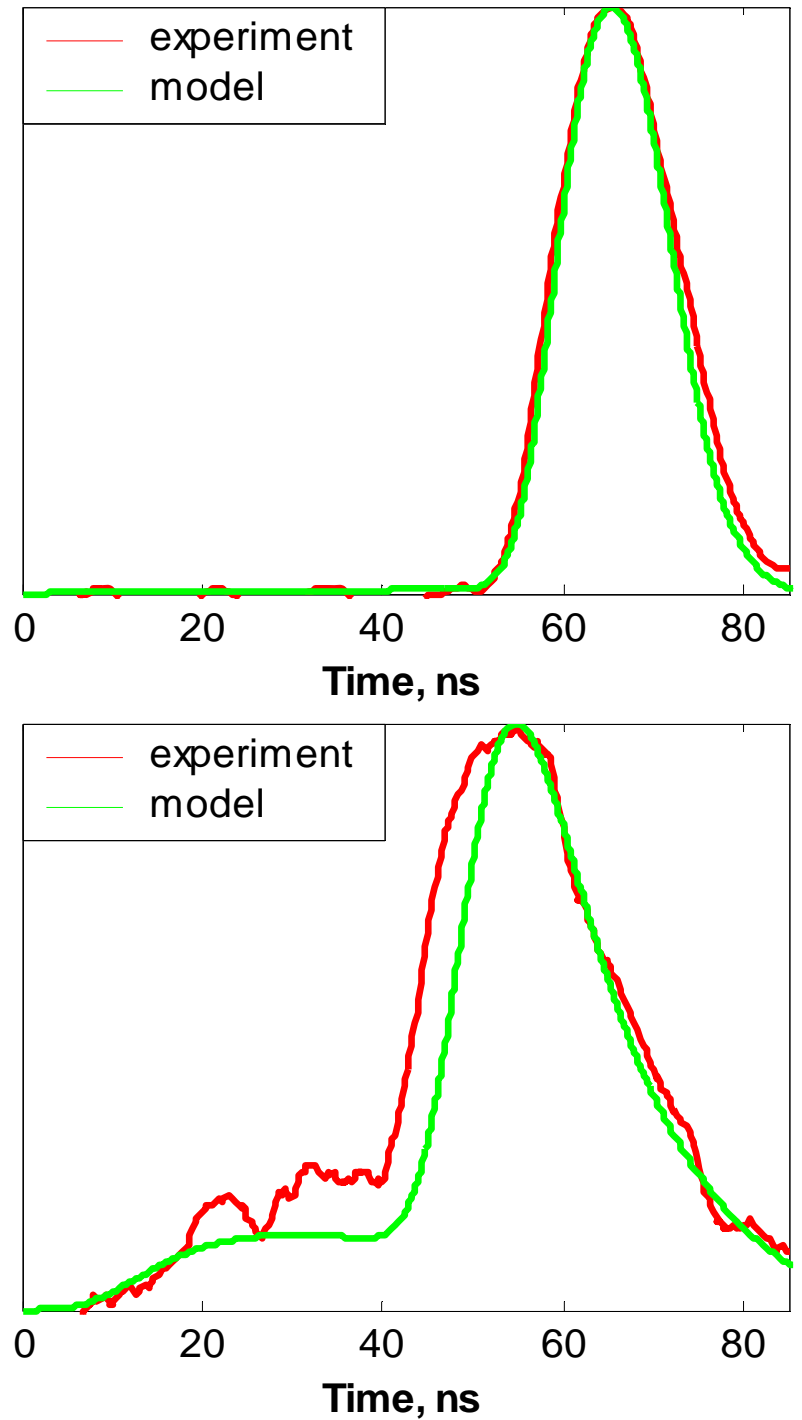

FIG. 8 Graphs of the experimental backscatter data (red) and model results (green) on arbitrary linear scale. Top: attenuation coefficient $c=0.06 \mathrm{~m}^{-1}$, S-R separation $=250 \mathrm{~mm}$, angular aperture $=10 \mathrm{mR}$. Bottom: $c=0.25 \mathrm{~m}^{-1}, \mathrm{SR}=83 \mathrm{~mm}$, angular aperture $=10 \mathrm{mR}$.

perimental verifications (see Figure 9). A detailed comparison is the subject of a future publication.

\section{IMAGE SIMULATION MODEL}

The total backscatter irradiance collected by the receiver is defined as the backscatter component (EBS). The effect of backscatter on an acquired image is a reduction in contrast and signal-to-noise ratio. Backscatter is independent of target reflectance and can be reduced by increasing the sourcereceiver separation, or decreasing the laser and receiver angular apertures. However, as the scattering particle concentration increases, multiple-scattered backscatter levels increase, eventually leading to the contrast limit for a CW-LLS system. Not all the light received by a LLS system that has been reflected from the target contains useful information about the region of target being scanned at that instant in time. The component of the received light which has made it to the target, but which has undergone scattering with particles and water molecules on the outgoing path can be defined as the for- 


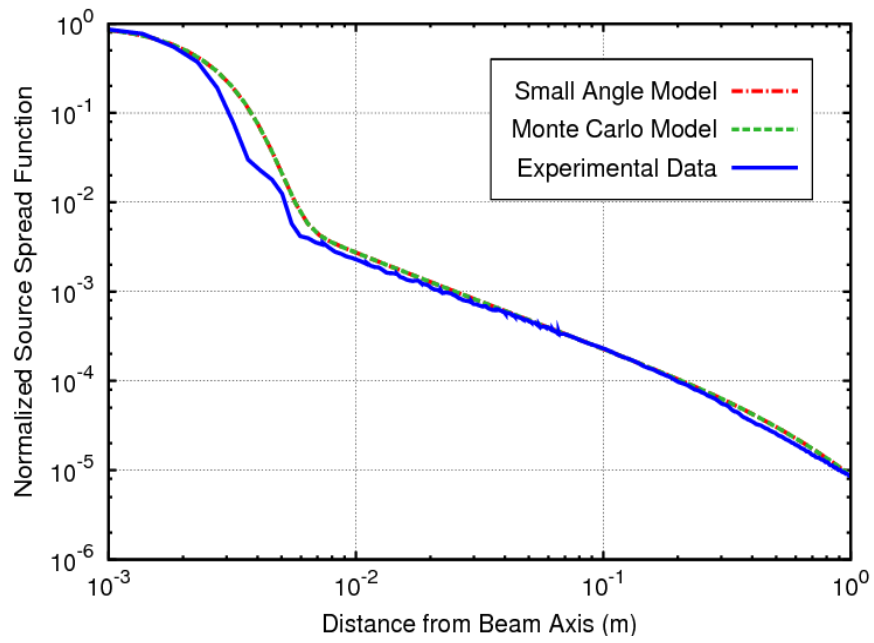

FIG. 9 Comparison of the normalized beam irradiance profile using Monte Carlo and small angle models with experimental measurements.

ward scattered component (EFS). Carrying reflectance information from a larger region of the target the main effect of forward scatter on an acquired image is a reduction in resolution, contrast and signal-to-noise ratio, particularly when the surrounding target has a high mean reflectance and forward scatter appears as a blurring or glow. For the non-coherent, direct detection LLS systems, the only way to minimize forward scatter is to reduce laser and receiver angular apertures. The image component that contains useful reflectance information from the small region of the target which is illuminated by the unscattered laser beam is called the direct component (ED). This consists of light which has not been scattered out of the main beam on the way to the target, but can consist of light which has undergone multiple small angle scattering on the way back from the target to the receiver, through those combinations of angles that allow acceptance into the receiver aperture.

It follows that each pixel formed by the CW-LLS consists of the linear superposition of all three components of returning light present at the receiver, i.e.

$$
E(x, y)_{\mathrm{CW}-\mathrm{LLS}}=E(x, y)_{\mathrm{BS}}+E(x, y)_{\mathrm{FS}}+E(x, y)_{\mathrm{D}} \text {. }
$$

The CW-LLS system will continuously integrate the total signal from the photomultiplier tube (PMT) over several microseconds for each image pixel. Longer integration times can improve signal to noise ratio when backscatter levels are high, but also lead to increased intra-pixel scan distance, and hence a reduction in spatial resolution.

Utilizing electronic gating to remove the backscatter component, each pixel formed by the PG-LLS consists of the linear superposition of only the direct and forward-scattered components of returning light present at the receiver, i.e.

$$
E(x, y)_{\mathrm{PG}-\mathrm{LLS}}=E(x, y)_{\mathrm{FS}}+E(x, y)_{\mathrm{D}} \text {. }
$$

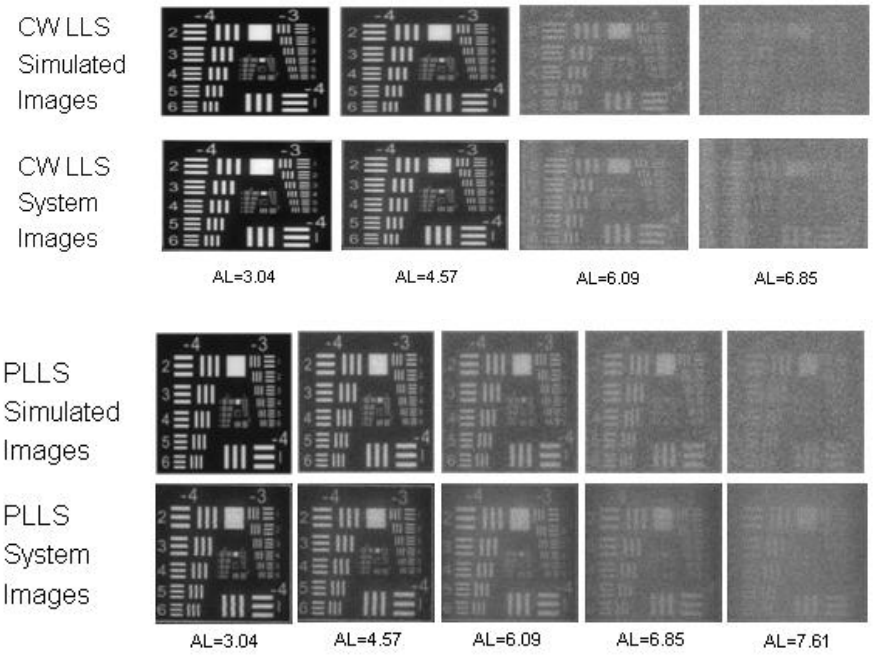

FIG. 10 (a) Simulated and measured imagery for a continuous wave laser line scan (CW-LLS) system are compared at multiple attenuation lengths $(\mathrm{AL}=c z)$ and a standoff distance $z=7 \mathrm{~m}$, (b) Simulated and measured imagery for a pulsed laser line scan (PLLS) system are compared at multiple attenuation lengths (AL) and a standoff distance $z=7 \mathrm{~m}$. Angular aperture $=4 \mathrm{mR}$.

\section{PERFORMANCE ANALYSIS: SIMULATION AND MEASUREMENT IMAGE COMPARISON}

In agreement with the EODES-3 model, recent test-tank work by the authors revealed significant imaging improvements were possible over the existing CW-LLS, by using a pulsedgated version of the LLS, known as PG-LLS [16]. The EODES3 LLS image simulation tool was used to investigate performance of the LLS technique as a function of beam attenuation length and standoff distance for a standard Air Force test chart. The test chart is input to the model and the simulator computes the resulting image response as a function of standoff distance, source-receiver separation, and optical attenuation among other parameters. The image simulation model has been validated and will be presented in a separate publication; however, for illustration of the model fidelity, we include a synoptic comparison of images obtained both experimentally and from the model in Figure 10. A detailed comparison would include a contrast measure for the various cases illustrated; however, a visual comparison shows adequate conformance of measured and simulated results given that imaging systems to date are generally interpreted by a "man-inthe loop". Of note here is that use of the pulsed laser system (PLLS) produces images at 7.61 attenuation lengths $(c z=7.61)$ that are visually equivalent to those produced by the CW-LLS system at 6.85 attenuation lengths. These images are produced using identical average laser power. The photomultiplier detector was gated on by an electronic gating circuit with a $30 \mathrm{~ns}$ delay from the time the laser pulse was emitted into the water tank.

\section{PERFORMANCE ANALYSIS: SIMULATED IMAGES FOR S-R SPACING}

Illustrated in Figure 11 are simulated images for 3 sourcereceiver separations of $5 \mathrm{~cm}, 20 \mathrm{~cm}$ and $40 \mathrm{~cm}$. The system 


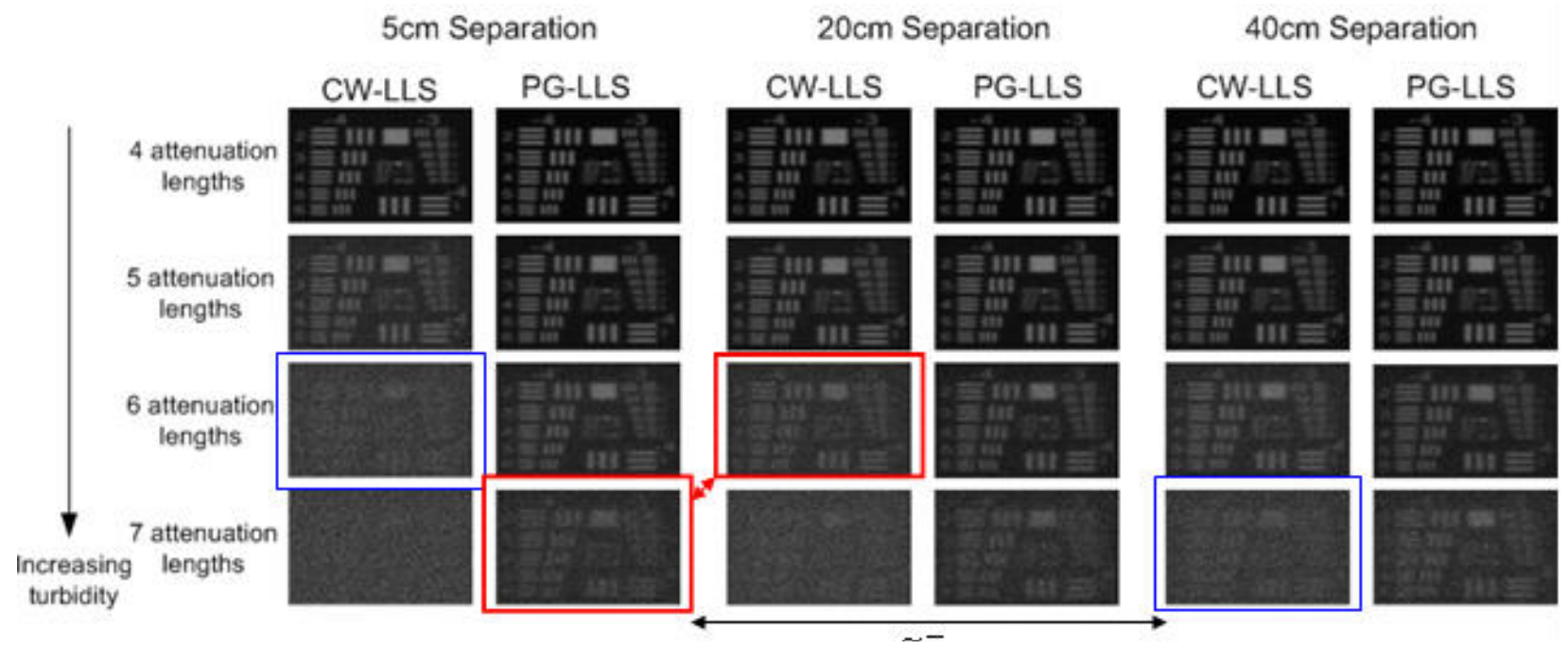

FIG. 11 Simulated technical target images demonstrating the expected turbid water performance of both CWLLS and PG-LLS payloads sized to be compatible with a variety of commonly used AUV form factors. A safe standoff distance of $7 \mathrm{~m}$ and a realistic cruising speed of $4 \mathrm{~ms}^{-1}$ are used. Receiver angular field is 4 mR (circular).

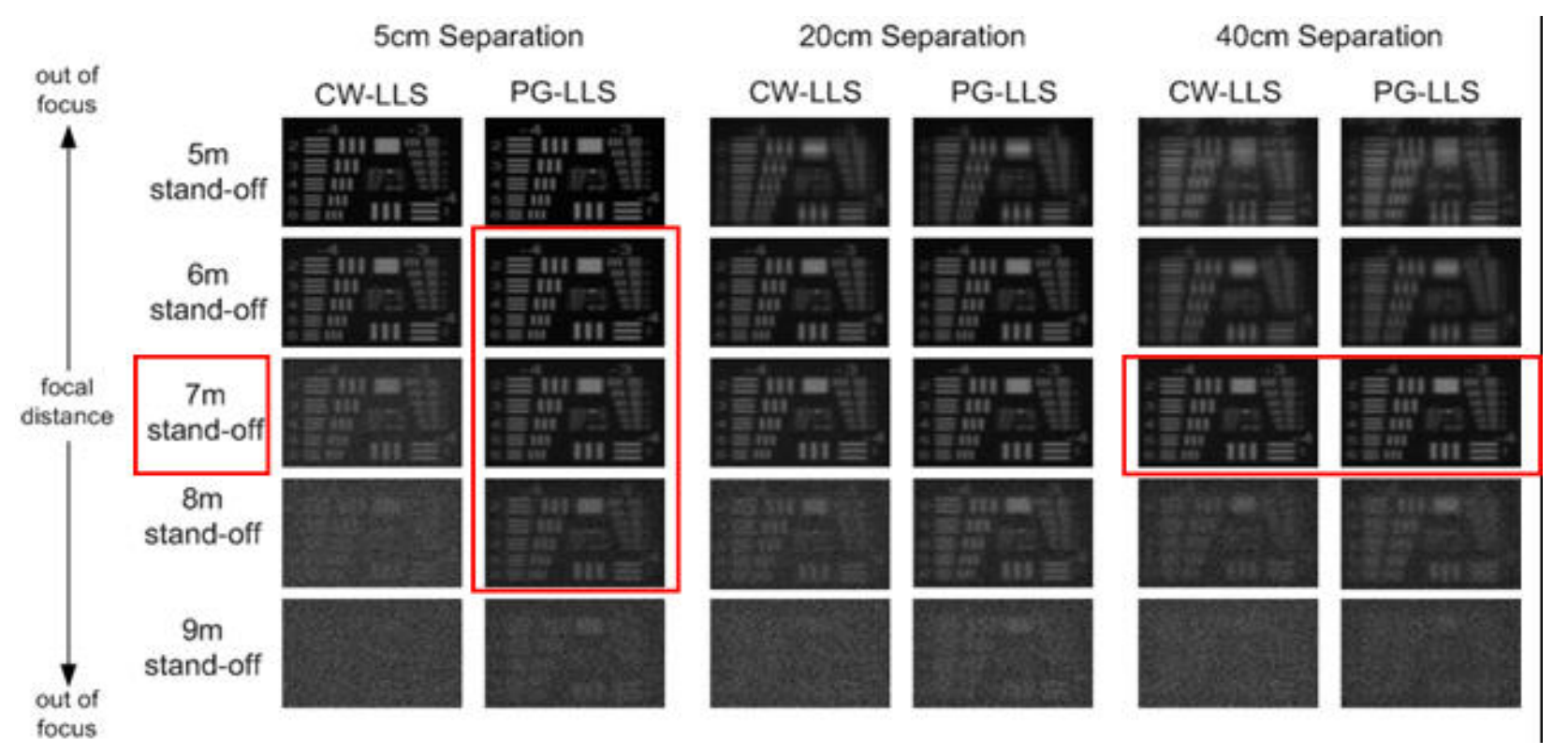

FIG. 12 Simulated technical target images at 5 beam attenuation lengths, focal distance of $7 \mathrm{~m}$ and platform speed of $4 \mathrm{~ms}^{-1}$ demonstrating the improved depth of field achievable from smaller $5 \mathrm{~cm}$ source-receiver separation in pulsed-gated LLS imaging system configurations.

model is configured for a nominal standoff distance $z=7 \mathrm{~m}$ and for a realistic line-to-line acquisition based a a vehicle speed of $4 \mathrm{~ms}^{-1}$.

The following observations can be made: (1) images for the PLLS system at 7 attenuation lengths $(c z=7)$ with $5 \mathrm{~cm} \mathrm{S-R}$ separation appear equivalent to the CW-LLS systems images at larger S-R separation of $20 \mathrm{~cm}$ (red outline), and (2) the CWLLS system can produce images at 7 attenuation lengths with $40 \mathrm{~cm}$ source-receiver separation, but if the separation is reduced to $5 \mathrm{~cm}$, the equivalent image contrast is obtained only to 6 attenuation lengths (blue outline), and (3) a $5 \mathrm{~cm}$ source receiver separation reaches a contrast limit at lesser standoff distance unless a pulsed-gated approach is adopted. The larger configurations do not show an obvious benefit of using a pulsed-gated scheme, and this likely explains one reason they have not been adopted in past larger deployed systems.

\section{PERFORMANCE COMPARISON: SIMULATED IMAGES AND DEPTH-OF-FIELD}

Simulated images were created using both LLS and PG-LLS systems configured for a nominal standoff distance of $7 \mathrm{~m}$. Images were then obtained for US Air Force test chart locations at $z=5 \mathrm{~m}, 6 \mathrm{~m}, 7 \mathrm{~m}, 8 \mathrm{~m}$, and $9 \mathrm{~m}$ standoff distances. All images results were then repeated for source-receiver separations of $5 \mathrm{~cm}, 20 \mathrm{~cm}$, and $40 \mathrm{~cm}$. All images are shown in Figure 12. Again, several results are apparent: (1) the $5 \mathrm{~cm} \mathrm{S-R}$ case shows greater depth-of-field (DOF) of $\pm 1 \mathrm{~m}$ for the PGLLS configuration compared to the CW-LLS case (leftmost red outline), (2) The larger configurations show strong image blurring and small DOF for either system (rightmost red outline). Reasons for this behavior are related to the geometric object misalignment with the receiver FOV for a given source angle, as can be inferred from Figure 1. 


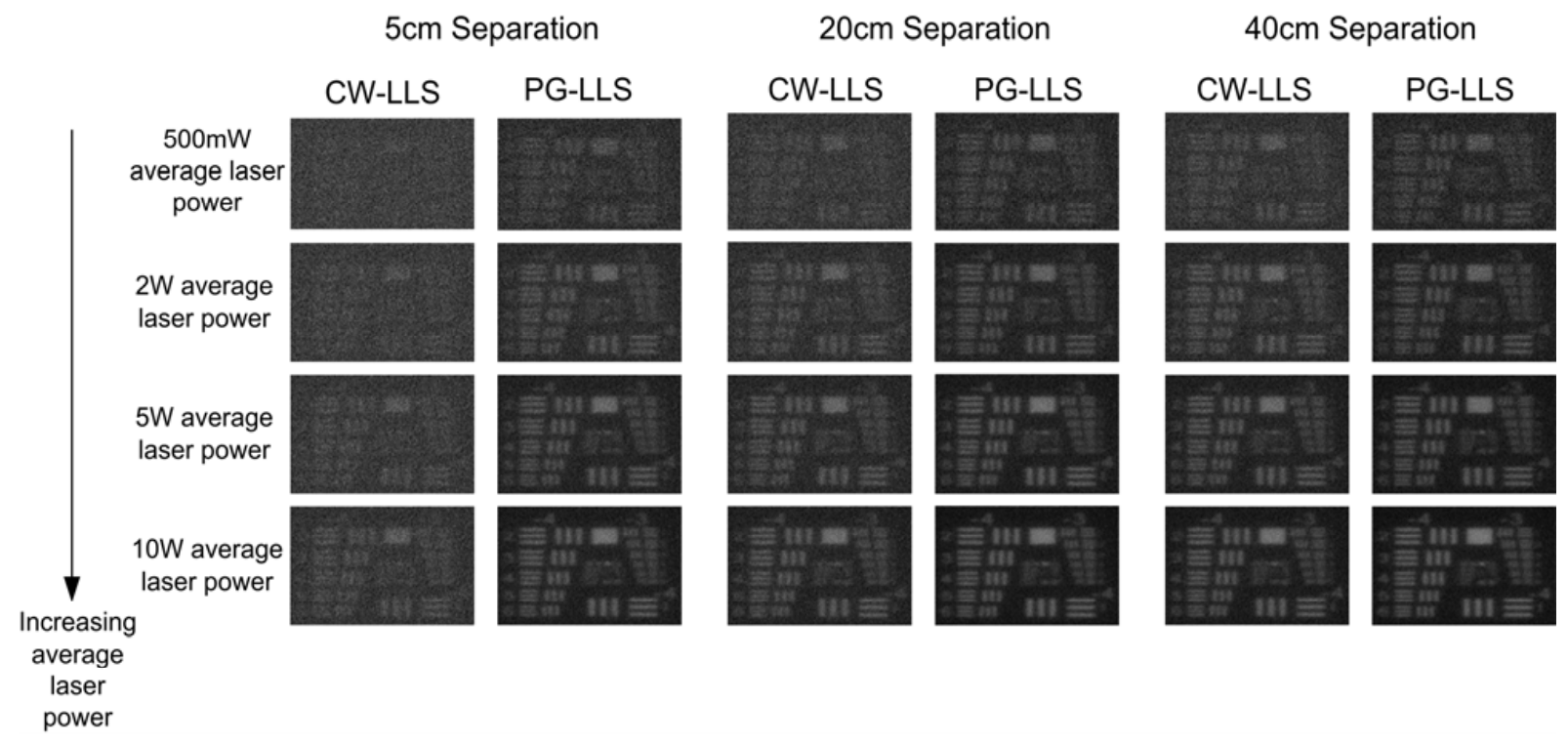

FIG. 13 Simulated technical target images demonstrating the expected turbid water performance at 6 beam attenuation lengths ( $c z=6$ ) versus average laser power for both CW-LLS and PG-LLS payloads sized to be compatible with a variety of commonly used AUV form factors. A safe stand-off distance $z=7 \mathrm{~m}$ and a realistic cruising speed of 4 ms ${ }^{-1}$ are used. Receiver angular field is $4 \mathrm{mR}$ (circular).

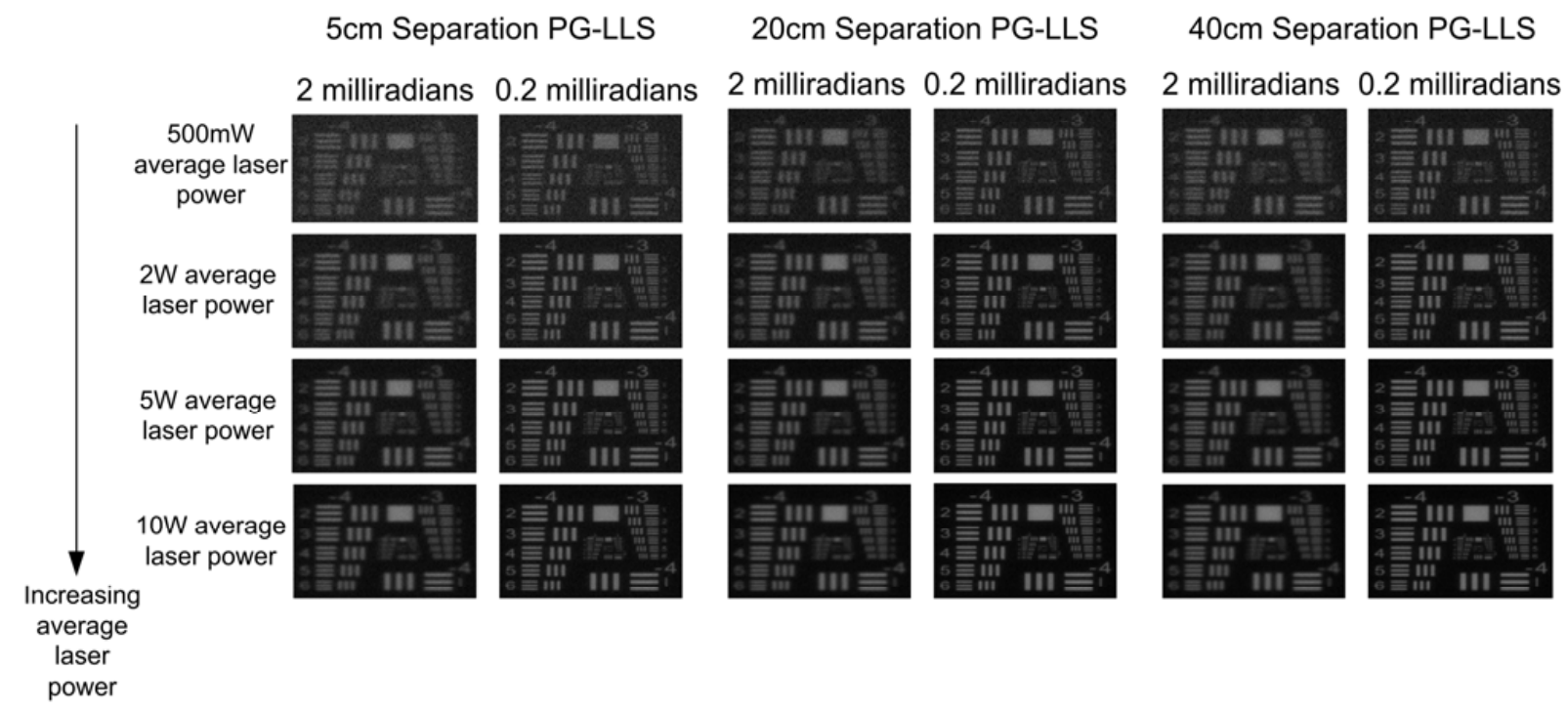

FIG. 14 Simulated technical target images examining the effect of laser beam divergence on turbid water imaging performance (at $c z=5$ beam attenuation lengths) for a PG-LLS system at source-receiver separations of $5 \mathrm{~cm}, 20 \mathrm{~cm}$, and $40 \mathrm{~cm}$. A safe stand-off distance $z=7 \mathrm{~m}$ and a realistic cruising speed of 4 ms ${ }^{-1}$ are used. Receiver angular field is $4 \mathrm{mR}$ (circular).

\section{PERFORMANCE COMPARISON: SIMULATED IMAGES FOR DIFFERENT SOURCE POWER}

Image contrast in these systems has generally been considered to be limited by the relatively small number of target photons returning to the detector in comparison to those arriving from scattering centers throughout the medium. Therefore, image contrast dependence on laser power is not expected to be particularly strong as long as sufficient photon numbers arrive from the target at a given standoff distance to overcome noise contributions in the detector circuitry. In Figure 13, we show the effects of laser power on the image contrast for a CW-LLS system in comparison to a PG-LLS system versus source-receiver separation. The following observations can be made: (1) increases in average laser power for the pulsed-gated approach shows a more marked improvement in image quality, and (2) the improvement is more significant for source-receiver separation of $5 \mathrm{~cm}$. This makes sense when one considers that an increase in output power will also result in a larger backscatter signal, as well as target signal, and that pulse gating reduces the backscatter contribution to the image background noise.

\section{PERFORMANCE COMPARISON: SIMULATED IMAGES FOR DIFFERENT SOURCE POWER AND LASER ANGULAR DIVERGENCE}

To examine the effects of laser divergence angle on image quality (resolution), simulations were conducted for two 
cases: $2 \mathrm{mR}$ and $0.2 \mathrm{mR}$. Simulated images were acquired for the PG-LLS system and the average power was varied from $500 \mathrm{~mW}$ to $10 \mathrm{~W}$. Results are shown in Figure 14. Generally, the higher power lasers produce better images and image resolution is improved for the smaller laser divergence angle for the chosen environmental conditions, $c z=5$ and $z=7 \mathrm{~m}$. These conditions are representative of typical coastal ocean environments.

\section{CONCLUSIONS}

The larger-scale motivation for this work is to investigate the use of scanned pulsed laser sources with gated receivers for more compact implementations of extended range underwater laser imaging systems than are currently available. An image simulation computer model, developed under the auspices of this and other programs [14] has been employed in developing the presented system and image comparisons. Using the simulation model, we illustrated the physical effects responsible for image degradation in synchronously scanned laser imaging systems, and have shown the potential advantages of using pulsed laser system configurations via simulated image comparisons.

For the more compact implementations with smaller sourcereceiver separations, which are more suited as payloads for man-portable UUVs, it is clear that the PG-LLS approach is necessary to allow for performance beyond 5 attenuation lengths. Furthermore, such a small separation makes the system more immune to rapid changes in altitude, as shown. Assuming the effectiveness of the gating mechanism is not affected by a larger backscatter return, the use of higher average power sources will further improve performance for the PGLLS case. Finally, the model shows that at operational ranges through 5 attenuation lengths, the use of a near diffractionlimited beam will yield higher resolution images than what is possible with beam angular divergence specifications typical of many lasers.

\section{ACKNOWLEDGEMENTS}

The authors would like to acknowledge the Steven Ackelson and the U.S. Office of Naval Research for oversight of this program, as well collaborators Joe Shirron and Tom Giddings of Metron, Inc., and Charles Mazel of Physical Sciences, Inc. for model development and analysis.

\section{References}

[1] M. P. Strand, Quantitative evaluation of environmental noise in underwater electro-optic imaging systems (Ocean Optics XIV, KailuaKona, Hawaii, 10-13 November 1998).

[2] T. J. Kulp, D. Garvis, R. Kennedy, T. Salmon, and K. Cooper, “Results of the final tank test of the LLNL/NAVSEA Synchronous-Scanning Underwater Laser Imaging System" Proc. SPIE 1750, 453-464 (1992).

[3] A. Gordon, Turbid test results of the SM200o laser line scan system and low light level underwater camera tests (Underwater In- tervention '94: Man and Machine Underwater, Marine Technology Society, Washington D.C., 305-311, 1994).

[4] M. P. Strand, “Underwater electro-optical system for mine identification" Proc. SPIE 2496, 487-497 (1995).

[5] A. Laux, L. J. Mullen, and B. Cochenour, A comparison of extended range laser line scan imaging techniques in turbid underwater environments (Ocean Optics XIX, II Ciocco, 6-10 October 2008).

[6] E. A. McLean, H. R. Burris, and M. P. Strand, "Short-pulse rangegated optical imaging in turbid water" Appl. Opt. 34, 4343-4351 (1995).

[7] B. A. Swartz, Diver and ROV deployable laser range gated underwater imaging systems (Underwater Intervention '93, Marine Technology Society and Association of Diving Contractors, New Orleans, 1993).

[8] N. H. Witherspoon, and J. H. Holloway, "Feasibility testing of a range-gated laser-illuminated underwater imaging system" Proc. SPIE 1302, 414 (1990).

[9] G. R. Fournier, D. Bonnier, J. L. Forand, and P. W. Pace, “Rangegated underwater laser imaging system" Opt. Eng. 32, 2185-2190 (1993).

[10] J. Busck, “Underwater 3-D optical imaging with a gated viewing laser radar" Opt. Eng. 44, 116001 (2005).

[11] F. M. Caimi, F. R. Dalgleish, T. E. Giddings, J. J. Shirron, C. H. Mazel, and $\mathrm{K}$. Chiang, Pulse versus $\mathrm{CW}$ laser line scan imaging detection methods: simulation results (IEEE/OES Oceans Oceans '07, Aberdeen, 18-21 June 2007).

[12] F. R. Dalgleish, F. M. Caimi, C. H. Mazel, J. M. Glynn, K. Chiang, T. E. Giddings, and J. J. Shirron, Model-based evaluation of pulsed lasers for an underwater laser line scan imager (Ocean optics XVIII, Montreal, 9-13 October 2006).

[13] F. R. Dalgleish, F. M. Caimi, C. H. Mazel, and J. M. Glynn, Extended range underwater optical imaging architecture (MTS/IEEE Oceans '06, Boston, 18-21 September 2006).

[14] T. E. Giddings, and J. J. Shirron, "Numerical simulation of the incoherent electro-optical imaging process in plane-stratified media" Opt. Eng. 48, 126001 (2009).

[15] F. M. Caimi, Private communication with Brian Coles (Raytheon, 1993).

[16] F. M. Caimi, and F. R. Dalgleish, Performance considerations for laser line scan (LLS) imaging systems (EOS Topical Meeting on "Blue" Photonics, Aberdeen, 18-19 August 2009).

[17] D. A. DeWolf, "Electromagnetic reflection from an extended turbulent medium: cumulative forward-scatter single-backscatter approximation" IEEE T. Antenn. Propag. 19, 254-262 (1971).

[18] J. Jaffe, New advances in underwater imaging: seeing faster, farther, better (EOS Topical Meeting on "Blue" Photonics, Aberdeen, 18-19 August 2009).

[19] L. S. Dolin, "Propagation of a narrow light beam in a random medium" Izv. VUZ Radiofiz.+ 7, 380-382 (1964).

[20] L. S. Dolin, and V. A. Savel'yev, "Backscattering signal in pulsed irradiation of a turbid medium with a narrow directional light beam" Izv. Atmos. Ocean Phy.+ 7, 328-331 (1971).

[21] L. E. Mertens, and F. S. Replogle Jr., "Use of point spread and beam spread functions for analysis of imaging systems in water" J. Opt. Soc. Am. 67, 1105-1117 (1977).

[22] V. A. Korshunov, "Laser radar equation in the small-angle approximation" Radiophys. Quantum El. 24, 748-755 (1980). 
[23] E. P. Zege, I. L. Katsev, and I. N. Polonsky, "Analytical solution to LIDAR return signals from clouds with regard to multiple scattering" Appl. Phys. B-Lasers 0. 60, 345-353 (1995).

[24] I. L. Katsev, E. P. Zege, A. S. Prikhach, and I. N. Polonsky, “Efficient technique to determine backscattered light power for various atmospheric and oceanic sounding and imaging systems" J. 0pt. Soc. Am. A 14, 1338-1346 (1997).

[25] T. E. Giddings, and J. J. Shirron, "Numerical simulation of the electro-optical imaging process in plane-stratified media" submit- ted to Appl. Opt. (2008).

[26] F. R. Dalgleish, F. M. Caimi, W. B. Britton, and C. F. Andren, "Improved LLS imaging performance in scattering-dominant waters" Proc. SPIE 7317, 73170E (2009).

[27] A. Laux, R. Billmers, L. Mullen, B. Concannon, J. Davis, J. Prentice, and V. Contarino, "The abc's of oceanographic lidar predictions: a significant step toward closing the loop between theory and experiment" J. Mod. Opt. 49, 439-451 (2002). 